\title{
RECONSTRUCTION OF THE THORACIC WALL WITH EPIPLOON AND DERMO-CUT ABDOMINAL LOWER NEO FLAP IN BREAST ANGIOSARCOMA - CASE REPORT
}

Maria Eduarda Bernardino Martins Melo', Darley de Lima Ferreira², Irnanda Layanna Gomes Oliveira', Julia Cavalcanti dos Santos Carneiro', Gabriela Prado Lopes ${ }^{1}$

${ }^{1}$ Centro Universitario Maurício de Nassau - Recife (PE), Brazil.

${ }^{2}$ Hospital Barão de Lucena - Recife (PE), Brazil.

Introduction: Breast angiosarcoma is very rare and highly aggressive. It has an incidence of $0.5 \%$ to $1 \%$ of cases and its presentation is typically in women between 14 and 82 years of age with an average age that revolves around 35 years. Breast angiosarcoma presents clinically as a unilateral, softened tumor with ill-defined borders and skin thickening, with a variable growth rate. However, if compared to epithelial breast cancer, angiosarcoma has a faster growth rate. The second is known as stromal sarcoma, fibrosarcoma, leiomyosarcoma, histiocytoma, and giant cell sarcoma. Regarding adjuvant therapy, chemotherapy and radiotherapy, they present an ineffective response. The effective treatment to offer a chance of healing is a broad surgical approach. Case report: Female patient U..S., 35, from Paulo Afonso, state of Pernambuco, complaining of a left breast nodule. She had a tumorectomy in her city, due to fibroadenoma and phyloid tumors with atypia and mitosis. In our service, she underwent a series of tests, where mammogram/ultrasonography confirmed the presence of a $1.5 \mathrm{~cm}$ nodule in the left breast.She underwent a new segmental resection surgery, whose histopathology confirmed a low-grade malignant cystosarcoma phyllodes and exiguous margins.A new surgical proposal was discussed with the patient - a simple mastectomy with immediate reconstruction with silicone breast implant and latissimus dorsi flap. The surgery was performed and the histopathological result was the absence of residual neoplastic tissue, with an area of scar fibrosis and usual ductal hyperplasia. After her recovery, the patient was referred to clinical oncology and radiotherapy, but both had no indication for adjuvant therapy. After one year, the patient returned to undergo symmetrization of the opposite breast and reconstruction of the nipple-areolar complex. After two years, the patient returned with a breast US, which demonstrated a nodular image of $1.5 \mathrm{~cm}$ adjacent to the breast prosthesis and an MRI suggested the same image. A core biopsy was performed, which confirmed a malignant variant recurrent tumor. The tumor evolved very quickly and the surgery was performed with an enlarged resection of the entire pectoralis large and small and skin inclusion. To correct the deformity, we used the rotation of the great epiploon with the lower abdominal dermocutaneous flap. 